\title{
Cognitive flexibility mediates the association between early life stress and
}

\section{habitual behavior}

Xinqi Zhou ${ }^{1}$, Yayun Meng ${ }^{2}$, Helena S. Schmitt ${ }^{1,3}$, Christian Montag ${ }^{1,3}$, Keith M. Kendrick ${ }^{1}$, Benjamin Becker ${ }^{1 *}$

${ }^{1}$ The Clinical Hospital of Chengdu Brain Science Institute, MOE Key Laboratory for Neuroinformation, University of Electronic Science and Technology of China, Chengdu, China

${ }^{2}$ Faculty of Teacher Education, Hechi University, Hechi, China

${ }^{3}$ Department of Molecular Psychology, Institute of Psychology and Education, Ulm University, Ulm, Germany

*correspondence to Benjamin Becker, PhD Center for Information in Medicine University of Electronic Science and Technology of China Chengdu 611731, China

Tel.: +862861 830811

Mail:ben_becker@gmx.de 


\begin{abstract}
Accumulating evidence suggests that exposure to high levels of early life stress (ELS) may lead to a lasting shift between goal-directed and habitual behavioral tendencies. Cognitive flexibility has been shown to be impaired following early life stress and represents a protective factor for the formation of rigid maladaptive behavior, however, whether cognitive flexibility mediates their association is not clear. Against this background we employed a mediation approach in a sample of $n=560$ young healthy Chinese to determine whether cognitive flexibility mediates the association between ELS and habitual behavioral tendencies as assessed by the Creature of Habits Scale (COHS). We present and validate a Chinese version of the COHS (COHS-C) and replicate the two factor solution of the original version. Higher ELS exposure was associated with higher habitual behavioral tendencies and lower cognitive flexibility. Importantly, the association between ELS and habitual behavior was fully mediated by cognitive flexibility, suggesting that ELS-associated deficient cognitive flexibility promotes habitual behavioral tendencies in everyday life. Early intervention approaches that aim at promoting cognitive flexibility may increase resilience for dysregulated habit formation following ELS in adulthood.
\end{abstract}

\title{
Keywords
}

Creature of Habit Scale, habitual tendencies, cognitive flexibility, early life stress, childhood maltreatment 


\section{Introduction}

Humans undergo prolonged periods of physical and mental development allowing them to adapt to challenging and complex environments. Together with genetic factors experiences during early sensitive periods lay the foundation for both, adaptive as well as maladaptive behavior during later adulthood. Aversive early life experiences such as childhood neglect or maltreatment can have lasting effects on cognition and behavior in later life (Sonuga-Barke et al., 2017). Accumulating evidence from different lines of research suggests that exposure to early life stress (ELS) associates with lower dispositional trust (Wu, Yuan, \& Kou, 2020), more aggression (Simpson, Griskevicius, Kuo, Sung, \& Collins, 2012), more suicidal ideation (Puzia, Kraines, Liu, \& Kleiman, 2014) and a strongly increased risk for the development of psychiatric disorders including anxiety, substance use and personality disorders during adulthood (Bonapersona, Joels, \& Sarabdjitsingh, 2018; Teicher \& Samson, 2016; Teicher, Samson, Anderson, \& Ohashi, 2016; Ten Have et al., 2019). Overarching theories have argued that the ELS-associated alterations may represent adaptations that promote survival in a malevolent environment (e.g. Gibb, Schofield, \& Coles, 2009; Teicher \& Samson, 2016; Teicher et al., 2016). For instance, faster automatic responses, elevated anxious arousal or lower trust may help to avoid harm in the context of childhood maltreatment, however, can become maladaptive during later adulthood and promote the development of psychiatric disorders. On the other hand, recently emerging findings from experimental studies have provided convergent evidence for lasting ELS-associated deficits in several cognitive domains with initial suggestions that ELS-associated alterations in implicit and reward-based learning as well as cognitive flexibility may represent a critical developmental pathway for the manifestation of psychopathological problems in later life (e.g. Harms, Shannon Bowen, Hanson, \& Pollak, 2018; Sheridan et al., 2018). However, considerable individual variations in the long-term detrimental effects of ELS have been reported, such that even after severe ELS part of individuals do not exhibit cognitive impairments or an elevated psychopathological load (Claessens et al., 2011; Daskalakis, Bagot, Parker, Vinkers, \& de Kloet, 2013; Enoch, 2011; Rutter, Kumsta, Schlotz, \& Sonuga-Barke, 2012; Sonuga-Barke et al., 2017).

Habits allow automatic responses in routine contexts and may facilitate appropriate responses under situations of limited cognitive capacity, e.g. due to acute or chronic stress. Habits represent learned behavioral responses that become automatically activated by associated cues or environments after a person repeatedly performs initially goal-directed actions in a specific situation (Wood \& Runger, 2016). Across the different research perspectives on habit formation, instrumental and reinforcement learning are considered as key mechanisms that promote the acquisition of habits during the course of repeated responding that ultimately form context- or stimulus-response associations in memory (Harms et al., 2018; Wood \& Runger, 2016). The habitual sequence of actions on the behavioral level, or as a mental representation, becomes activated by the corresponding cue or environment and the initially goal-directed behavior becomes under control of the stimulus-response association, rendering the behavior insensitive to changes in the value of the outcome (Harms et al., 2018; Wood \& Runger, 2016). Although habits can relieve cognitive load and facilitate fast responses during challenging situations, dysregulated habit 
formation can promote maladaptive behaviors that are hypothesized to underlie the development and maintenance of substance use disorders or obsessive-compulsive disorder (Everitt \& Robbins, 2016; Robbins \& Costa, 2017). Given that during the process of habit formation control over behavior gradually shifts away from goal-directed intentions towards associated stimuli, habits are difficult to change even in the context of intentional efforts or knowledge of the negative consequences of habitual actions (Ersche et al., 2016; Wood \& Runger, 2016). For instance, in the context of substance use disorders the transition from initial recreational to addictive use has been conceptualized as dysregulated habit formation promoting the transition from initially impulsive substance use to habitual and ultimately compulsive use (Everitt \& Robbins, 2016; Zhou et al., 2019). This transition may explain continued drug use despite a lack of pleasurable drug effects and severe detrimental effects.

Substantial experimental evidence suggests that stress induces a shift from goal-directed to habitual behavior (Schwabe \& Wolf, 2011), and exposure to ELS strongly increases the likelihood of developing an alcohol or illicit substance use dependence (Bonapersona et al., 2018; Enoch, 2011). Furthermore, neuroimaging studies have demonstrated robust alterations in fronto-striatal circuits in individuals with substance use disorders (KlugahBrown et al., 2020) and the identified circuits partly overlap with brain systems that mediate habit formation and ELS-induced neural plasticity (Dias-Ferreira et al., 2009; Harms et al., 2018; Teicher \& Samson, 2016; Teicher et al., 2016; Uhart \& Wand, 2009). A recent study that examined associations between variations in self-reported habitual behavior by means of the newly developed Creature of Habits Scale (Ersche, Lim, Ward, Robbins, \& Stochl, 2017) revealed initial evidence that subjects with higher levels of ELS reported more habitual behavior in daily life as compared to subjects with low aversive early life experiences.

Together with a recent experimental study reporting that higher levels of ELS are associated with increased avoidance habits on the behavioral level (Patterson, Craske, \& Knowlton, 2019), these findings suggest that ELS could shift the balance between goal-directed and habitual behavior in favor of the latter.

However, the precise pathways by which ELS could induce the shift from goal-directed to habitual behavior remain unclear. On the one hand stronger formation of habits may have beneficial and survival-promoting effects in a malevolent environment, on the other hand it is conceivable that stronger reliance on habits may evolve as a consequence of impaired goal-directed behavior due to chronic stress during early developmental periods. The successful implementation of goal-directed behavior and flexible adaptation to changes in the outcome of behavioral responses requires (among other functions) cognitive flexibility, that is the ability to switch cognitive strategies to adapt to changing environments. High levels of cognitive flexibility have been increasingly demonstrated to be a protective factor that may help individuals to adaptively cope with stressful life events (Murphy, Michael, \& Sahakian, 2012). More rigid and less flexible responses have been observed in individuals following exposure to high levels of ELS, such that these individuals had an impaired ability to switch from learned behavioral patterns to new action sequences (Harms et al., 2018). 
Against this background the present study examined a mediation model to determine relationships between ELS, cognitive flexibility and habitual behavior in everyday life (Fig.1). In our model we specified cognitive flexibility as a potential mediator which could mediate the impact of ELS on individual variations in habitual behavior, either partly or totally. According to an overarching theory proposed by (Dennis \& Vander Wal, 2010), cognitive flexibility consists of the tendency to perceive situations as controllable, the ability to perceive multiple alternative explanations and the ability to generate multiple alternative solutions when encountering challenging situations. Based on this multi-dimensional nature of cognitive flexibility, the authors developed the Cognitive Flexibility Inventory (CFI), a selfreport measure that assess the three dimensions on separate subscales (Dennis \& Vander Wal, 2010). The recently developed and evaluated Creature of Habit Scale (COHS) represents a validated self-report measure to assess individual variations in the extent of habitual behavior in everyday life (Ersche et al., 2017). Importantly, the previous study by Ersche et al. (2017) already showed that individuals with higher ELS exposure reported more habitual behavior in everyday life as compared to subjects with lower ELS exposure.

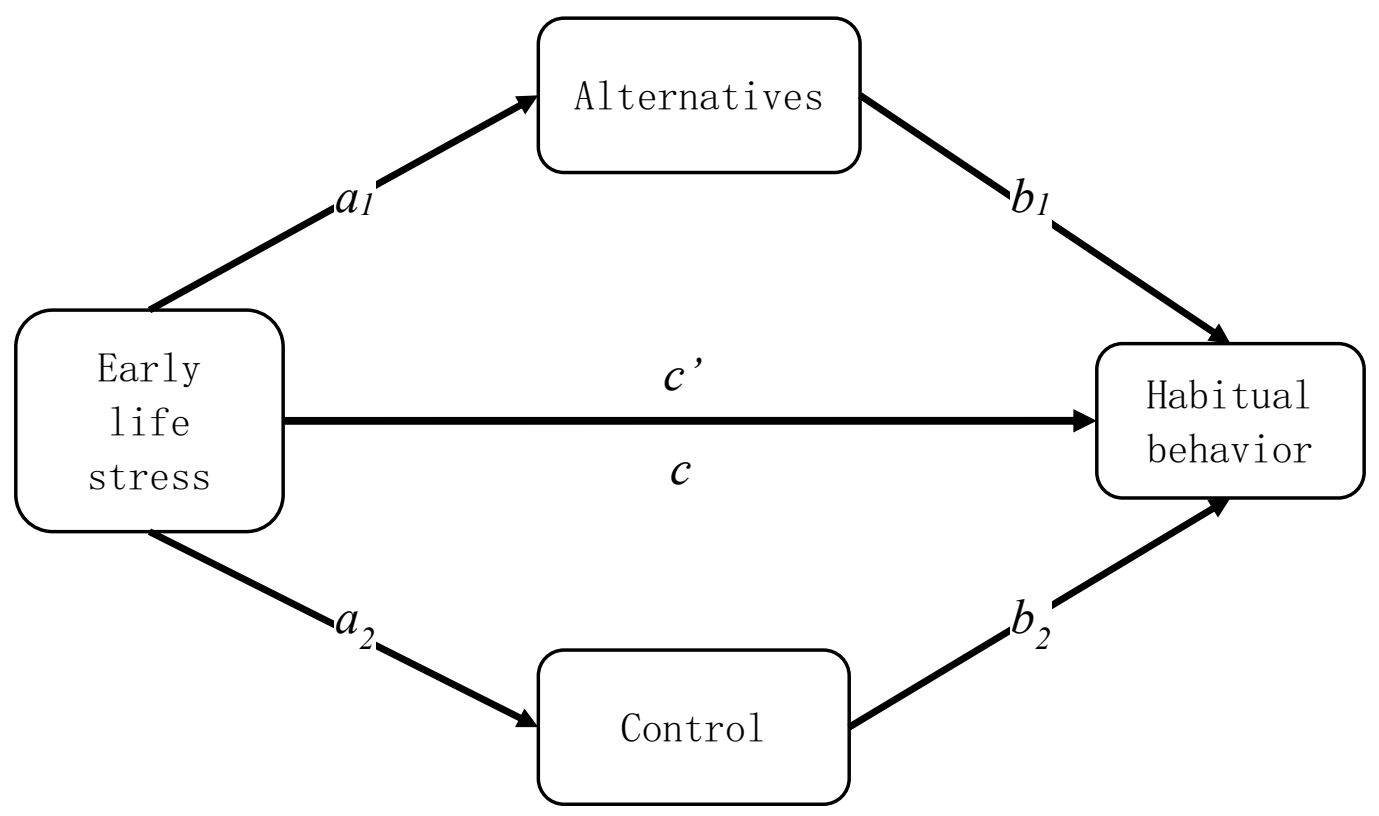

Fig.1 Hypothesized mediation model between early life stress, cognitive flexibility (two subscales: alternatives and control) and habitual behavior. Lowercase letters correspond to path coefficients.

In line with our hypotheses the aims of the current study were (1) to confirm the reliability and validity of a Chinese version of the COHS (COHS-C), (2) to investigate whether exposure to early life stress also increases self-reported habitual tendencies in Chinese participants, and (3) to explore whether this association is mediated by cognitive flexibility. Based on the 
previous literature we formulated a mediation model (Fig 1 ) to specifically test the following hypotheses $(\mathrm{H})$ :

(H1) Higher levels of ELS associate with higher levels of habitual behavior in the Chinese sample (in line with initial findings from Ersche et al., 2017).

(H2) Higher levels of ELS associate with lower cognitive flexibility (in line with initial findings from Harms et al., 2018).

(H3) Higher cognitive flexibility associates with lower habitual behavior.

$(\mathrm{H} 4)$ The mediation effect of cognitive flexibility on the association between higher ELS and habitual behavior is significant.

\section{Methods}

\subsection{Participants}

In line with previous studies on the original COHS (Ersche et al., 2017; Ersche et al., 2019) the assessments were conducted via an online platform (Chinese online survey platform, https://www.wjx.cn) and the number of subjects for the present study was based on the original COHS studies ( $N=362,533$ respectively in the original studies). A total of $N=647$ individuals from the Chinese college community, whose identity remained anonymous to the research team, participated in the online data collection. Participants were required to be $>18$ years old and Chinese (native-speakers) and free of a current or a history of a mental disorder. Participants from both sexes were included, and no restrictions with respect to employment or relationship status were imposed. As recommended by Meade and Craig (2012) an attention check item was included to identify careless participants. Additional items assessed current or history of mental disorders and medication use to validate the exclusion criteria. 75 participants (12\%) were excluded due to either invalid responses on the attention check item or incomplete data. 12 participants (2\%) who reported a current or history of a mental disorder or intake of medication were additionally excluded. The final sample for all subsequent analyses consisted of $\mathrm{N}=560$ participants ( $38 \%$ male) with a mean age of 19.6 years \pm 1.1 standard deviation (SD). In line with the aims of the present study all participants were administered the COHS-C (details see 2.2. Questionnaires), the Childhood Trauma Questionnaire (CTQ) (Bernstein et al., 2003) and the Cognitive Flexibility Inventory (CFI) (Dennis \& Vander Wal, 2010). The study procedures had full approval by the local ethics committee.

\subsection{Questionnaires}

\subsubsection{Childhood trauma questionnaire and Cognitive Flexibility Inventory}

To determine associations and mediation effects of ELS and cognitive flexibility with habitual behavior the CTQ and the CFI were administered. The CTQ represents a validated measure to assess the level of exposure to aversive experiences during early life. The CTQ includes 28 self-report items spanning five kinds of aversive experiences (emotional abuse, physical abuse, sexual abuse, emotional neglect, physical neglect) before the age of 16 (Bernstein et al., 2003) by means of five-point Likert scales. In line with previous studies the CTQ total score was used (Ersche et al., 2017; Liu et al., 2019). The total score can range from 25 to 125 , with higher scores reflecting higher exposure to ELS. Internal consistency in terms of 
Cronbach's alpha and McDonald's omega of the Chinese CTQ version (Zhao et al., 2005) version in the present sample were excellent $(\alpha=0.904$ and $\omega=0.944)$.

Cognitive flexibility was assessed by the previously validated $\mathrm{CFI}$, which requires participants to rate 20 self-report terms on a five-point Likert scale (Dennis \& Vander Wal, 2010). The CFI includes two subscales to measure three facets of cognitive flexibility: control subscale, 1) the tendency to perceive difficult situations as controllable, and alternatives subscale measures, alternatives subscale, 2) the ability to perceive multiple alternative explanations for life occurrences and human behavior, and 3) the ability to generate multiple alternative solutions to difficult situations. Higher scores indicate higher cognitive flexibility with respect to alternatives or control. The Cronbach's alpha and McDonald's omega of the two subscales from the Chinese version (Wang, Yang, Xiao, \& Su, 2016) were good to excellent in the present sample (alternatives: $\alpha=0.936, \omega=0.937$; control: $\alpha=0.862, \omega=0.869$ ).

\subsubsection{Translation of the original $\mathrm{COHS}$ including an introduction of the scale}

The original version of the Creature of Habit Scale (COHS) includes 27 items categorized into the two subscales automaticity ( 11 items) and routine ( 16 items). Both scales capture habitrelated behaviors given that they assess implicit behaviors that are often initiated without conscious awareness and goal-directed intention, however they demonstrate only weak correlations and have been proposed to differ with respect to their function and control over behavior (Ersche et al., 2017). Specifically, automaticity encompasses automatic responses triggered by specific contexts, whereas the routine subscale encompasses a series of familiar action patterns that involve regularity and are likely to be implemented on a daily basis (Ersche et al., 2017). Participants state their level of agreement for each of the 27 items on a 5-point Likert scale ranging from 1 (strongly disagree) to 5 (strongly agree). For both subscales, higher total scores indicate higher levels of automaticity or routine behavior, respectively. Previous studies demonstrated a good reliability and validity of the original COHS in healthy adults ( $>18$ years, $>98 \%$ native English speakers, $>79 \%$ Caucasian) from the United States of America (Ersche et al., 2017; Ersche et al., 2019). The two subscales exhibited good to excellent internal consistency reliability as assessed by Cronbach's alpha and McDonald's omega (routine: $\alpha=0.89-0.90 ; \omega=0.92-0.94$; automaticity: $\alpha=0.86-0.87$; $\omega=0.91-0.92$ ).

For the first step of translation, the original English version of the COHS was obtained from the Appendix B of Ersche et al. (2017). Next, a systematic forward-backward translation of the COHS from the original English version to Chinese followed the decentered translations strategy to retain the equivalency of meanings across the two languages and cultural settings (Huang, Hsueh, Lu, \& Huang, 2016). This procedure followed the principle of crosscultural adaptation of self-report measures for different languages (Beaton, Bombardier, Guillemin, \& Ferraz, 2000). Two native Chinese speakers with excellent English language proficiency, psychology background and previous experience in questionnaire translation each conducted an independent forward translation of the COHS into (simplified) Chinese. Subsequently, discrepancies between the two translated versions were thoroughly discussed and amended to produce the most appropriate and adequate translation (Peters \& 
Passchier, 2006). The back translation was conducted by a native Chinese-speaking psychological research assistant with a University degree in English language and linguistics who was blinded for the original English version of the COHS. Finally, the back-translated version and the original version were compared by one of the authors (H.S.S. psychology $\mathrm{PhD}$ student and proficient in English) to ensure literal and conceptual equivalence and to address discrepancies. All remaining discrepancies were resolved via thorough discussions among the authors and translators to ensure conceptual and semantic equivalence with the original version of the COHS. The preliminary version of the Chinese COHS (COHS-C) was evaluated for readability and comprehensibility by five college students (all native Chinese speakers). Subsequent interviews and discussions with the five participants resulted in only minor modifications to the final Chinese version of the COHS (COHS Chinese version, COHSC, detail items in supplementary table S1).

\subsection{Data analysis}

Statistical analyses were conducted using IBM SPSS Statistics Version 23 (IBM Inc., USA) and JASP (Jeffreys's Amazing Statistics Program, https://jasp-stats.org/), an open-source statistical software based on the R package (https://www.r-project.org/) . We conducted confirmatory factor analysis (CFA) to verify that the COHS-C structure consists of two latent factors (automaticity and routine) as reported in previous studies (Ersche et al., 2017; Ersche et al., 2019). The $\chi^{2}$ test, $\chi^{2} / d f$ ratio, root mean square error of approximation (RMSEA), standardized root mean square residual (SRMR), comparative fit index (CFI), goodness-of-fit index (GFI), Tucker-Lewis index (TLI) were used to estimate the construct validity and determine a model fit for the CFA which provides a robust maximum likelihood estimation. Internal consistency reliability of the subscales was assessed by McDonald's omega (McDonald, 2013) and Cronbach's alpha (Cronbach, 1951), with values of more than 0.70 considered as acceptable (Nunnally, 1994).

To avoid a common methodological bias, which leads to common variance due to the measurement method or the instrument - rather than the actual predispositions of individuals that the measurements are thought to examine - we performed Harman's signal factor test on all items of the current study (Podsakoff, MacKenzie, Lee, \& Podsakoff, 2003). In the present study we obtained 13 factors whose eigenvalues were greater than 1 , determined by exploratory factor analysis with unrotated factor solution, that totally accounted for $64.38 \%$ variances of all variables. The first single factor only accounted for $18.38 \%$ variance, which is less than one quarter of the variance in a typical research measure, arguing against the presence of significant measurement errors such as common method bias. Next, we estimated Pearson's correlation coefficients between the major variables in the present study: COHS-C routine and automaticity; CTQ total score; and, CFI alternatives and control. We additionally assessed the hypothesized mediation model (see Fig. 1) to determine the mediation effect of cognitive flexibility (alternatives and control) on the association between childhood adversity (CTQ) and habitual behavior (COHS-C automaticity and routine, respectively) by routines implemented in the PROCESS (Preacher \& Hayes, 2004) macro for SPSS. 95\% confidence internals (Cls), based on bias-corrected bootstrapping with 5,000 permutations, and Sobel test were used to test the significance of 
indirect effects (Preacher \& Hayes, 2008). For all analyses, $p$ values of less than 0.05 were considered statistically significant. Corresponding correlation and mediation analyses were additionally conducted with JASP to further validate the robustness of the results. To control for potential age and gender effects the mediation model was additionally recomputed including these variables as covariates.

\section{Results}

3.1 Reliability and Validity of the COHS-C

The two-factor structure fitted the data $\left(\chi^{2}=1169.33, \mathrm{df}=323, \mathrm{p}<0.001, \chi^{2} / d f=3.62\right.$, RMSEA $=0.068,90 \% \mathrm{Cl}$ for RMSEA $=[0.064,0.073), \mathrm{SRMR}=0.063, \mathrm{CFI}=0.799, \mathrm{GFI}=0.859$, $\mathrm{TLI}=0.781$, mean factor loading $=0.659$, factor loading range $=0.456-0.953)$, supporting the notion of automaticity and routine being two subscales of COHS-C. Both factors were also moderately correlated with each other $(r=0.575, p<0.001)$. Item loadings on both factors were high and significant, indicating high factorial validity of the items. Likewise, reliability of the coefficients for both factors was high as well, i.e. COHS routine $(\alpha=0.841 ; \omega=0.843$ ) and COHS automaticity ( $\alpha=0.844 ; \omega=0.849$ ), providing further support for satisfactory measurement precision of both subscales.

3.2 Correlations between early life stress, cognitive flexibility, and habitual behavior Early life stress as measured by the CTQ, was significantly negatively correlated with the control subscale of the CFI $(r=-0.323, p<0.001)$ and positively associated with both COHS scales assessing habitual behavior (routine, $r=0.147, p<0.001$; automaticity; $r=0.147, p<$ 0.001). However, there was no significant relationship between early life stress and alternative, as well as alternative and automaticity. Details are provided in table 1.

Table 1 Correlations between CTQ, CFI, and COHS-C.

\begin{tabular}{|c|c|c|c|c|c|c|}
\hline & Mean (SD) & CTQ & Alternatives & Control & Routine & Automaticity \\
\hline CTQ & $40.53(13.08)$ & - & & & & \\
\hline Alternatives & $46.16(8.71)$ & -0.044 & - & & & \\
\hline Control & $22.86(4.80)$ & $-0.323 * * *$ & $0.234^{* * *}$ & - & & \\
\hline Routine & $53.47(10.41)$ & $0.147 * * *$ & $0.174^{* * *}$ & $-0.301 * * *$ & - & \\
\hline Automaticity & $33.48(9.03)$ & $0.147 * * *$ & 0.039 & $-0.334 * * *$ & $0.575^{* * *}$ & - \\
\hline
\end{tabular}

3.3 Mediation effects of cognitive flexibility on the association between early life stress and habitual behavior

The mediation model used in this study revealed a significant total indirect effect of cognitive flexibility on the relationship between ELS and routine (Table 2). However, not all aspects of cognitive flexibility showed significant indirect effects. The confidence intervals for the indirect effect of alternatives encompassed zero, suggesting that alternatives was not a significant mediator of the effect of ELS on routine, and results were further confirmed by the Sobel test. Furthermore, there was no significant direct effect in this model (Fig. 2), suggesting a total mediation effect. 
Interestingly, we observed a similar pattern for the automaticity facet of habitual behavior, reflected by a total indirect effect of cognitive flexibility on the relationship between ELS and automaticity (Table 2). Only the indirect effect of the control facet of cognitive flexibility was significant, which was further validated by the results from the Sobel test. Moreover, the control facet was a total mediator of the association between ELS and automaticity (Fig. 3).

Summarizing, the level of cognitive flexibility, specifically the control facet, mediated the positive relationship between ELS and habitual behavior (routine and automaticity).

Specifically, early life stress had a negative correlation with the level of cognitive flexibility (control facet), which was strongly negatively associated with habitual behavior, for both the routine and automaticity facet (Fig. 2, 3). In additional analyses including sex and age as confounding variables findings from the two mediation models remained stable.

Table 2 Indirect effects of mediation models

\begin{tabular}{ccccccc}
\hline Paths & Effect & $\begin{array}{c}\text { Boot } \\
\text { SE }\end{array}$ & $\begin{array}{c}\text { Boot } \\
\text { LLCl }\end{array}$ & $\begin{array}{c}\text { Boot } \\
\text { ULCl }\end{array}$ & $z$ & $p$ \\
\hline CTQ $\rightarrow$ alternatives $\rightarrow$ routine & -0.009 & 0.0124 & -0.0364 & 0.0141 & -1.0202 & 0.3077 \\
CTQ $\rightarrow$ control $\rightarrow$ routine & 0.0887 & 0.019 & 0.0549 & 0.1295 & 5.7103 & $<0.001$ \\
Total & 0.0796 & 0.0251 & 0.0356 & 0.1347 & - & - \\
CTQ $\rightarrow$ alternatives $\rightarrow$ automaticity & -0.0037 & 0.0054 & -0.0183 & 0.0046 & -0.942 & 0.3462 \\
CTQ $\rightarrow$ control $\rightarrow$ automaticity & 0.0779 & 0.0167 & 0.0475 & 0.1139 & 5.6996 & $<0.001$ \\
Total & 0.0742 & 0.0184 & 0.0424 & 0.1145 & - & - \\
\hline
\end{tabular}

Boot SE: Bootstrap for Standard Error; LLCI: lower-level confidence interval; ULCI: upper-level confidence interval. 


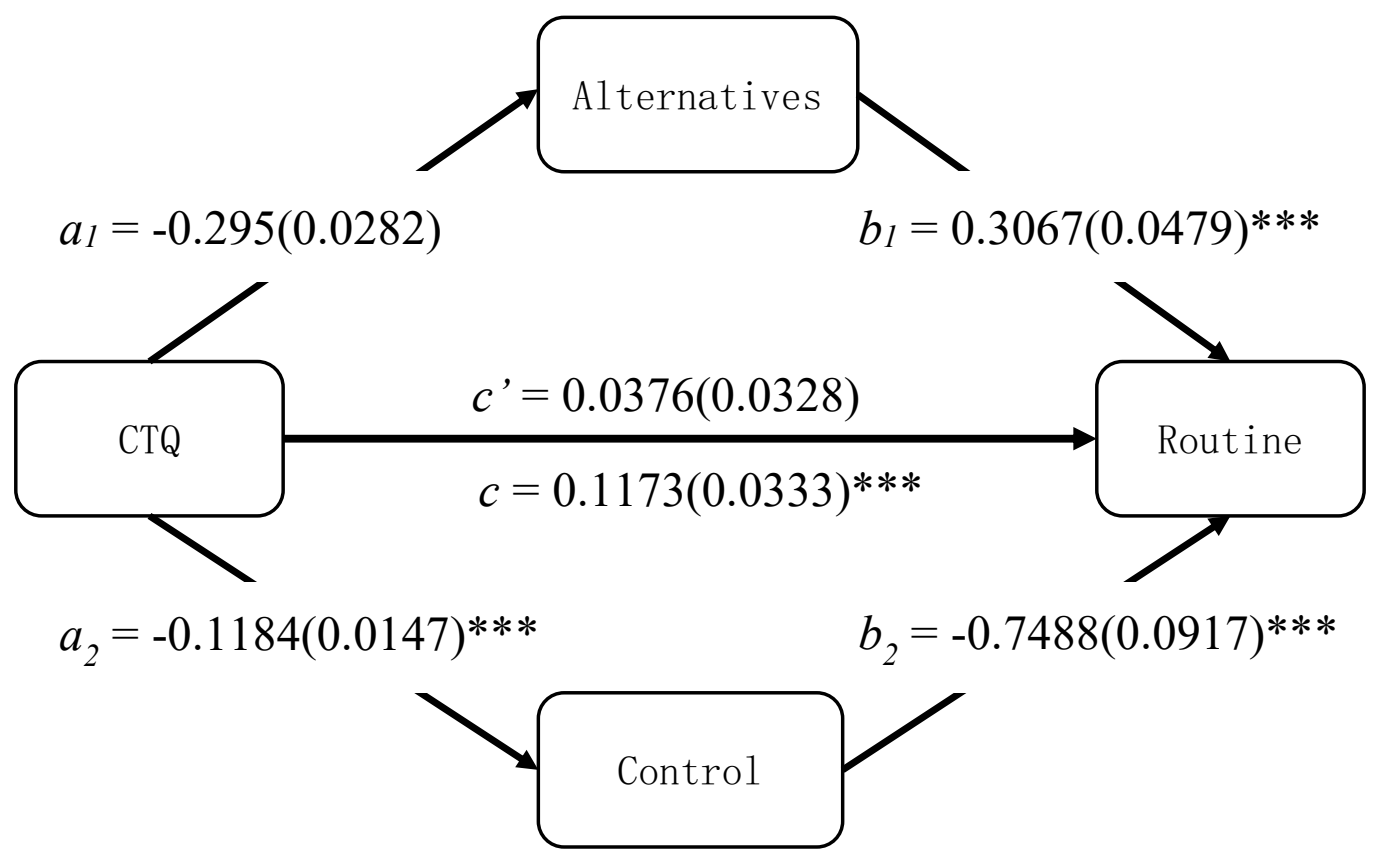

Fig. 2. Mediation model: mediation effect of cognitive flexibility on the relationship between early life stress (CTQ) and routine (COHS). Unstandardized coefficients are presented with standard errors in parentheses. $c^{\prime}$ : direct effect, $c$ : total effect, $* p<0.05, * * p<0.01, * * * p<0.001$. CTQ: Childhood Trauma Questionnaire. 


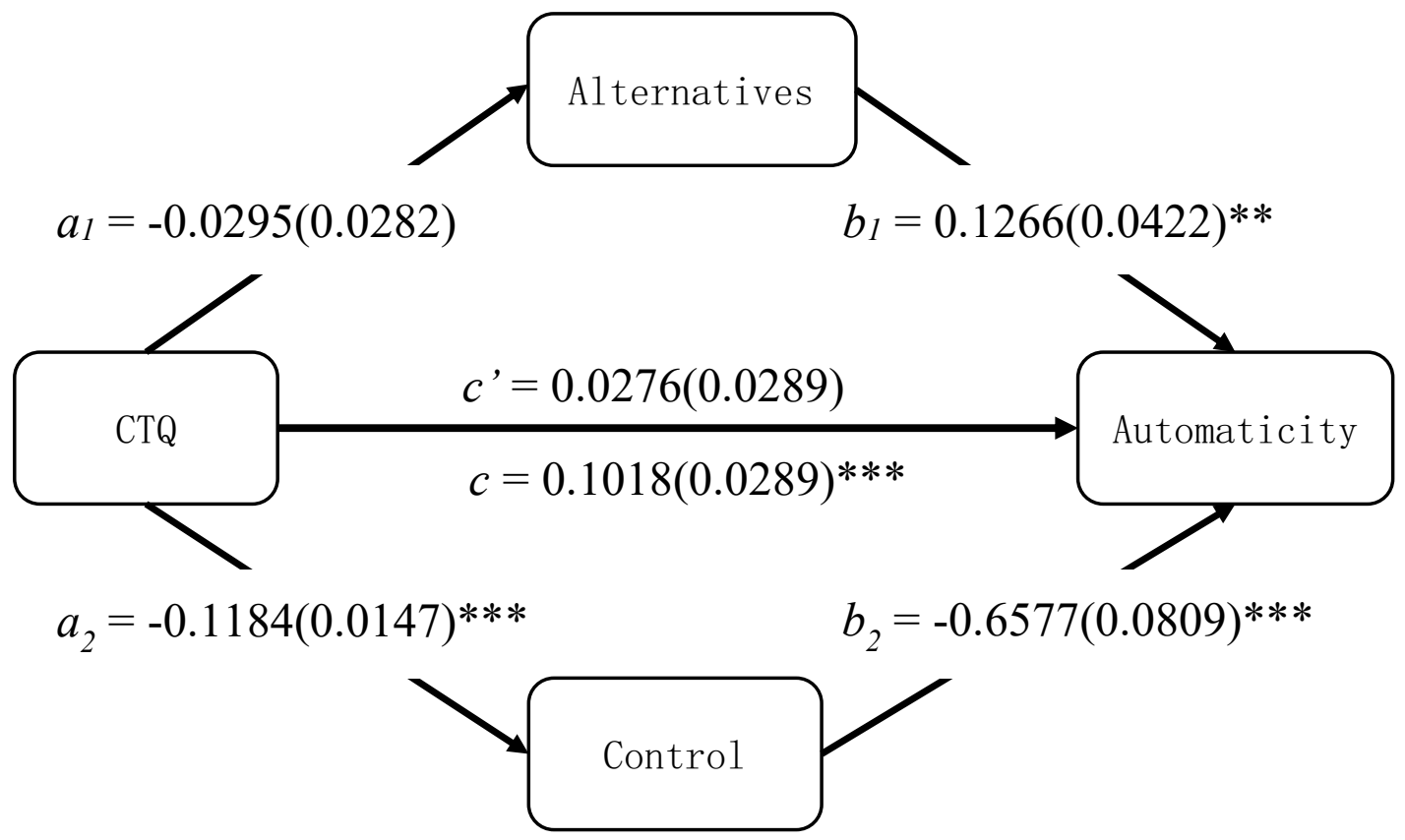

Fig. 3. Mediation model: mediation effects of cognitive flexibility on the relationship between early life stress (CTQ) and automaticity (COHS). Unstandardized coefficients are presented with standard errors in parentheses. $c^{\prime}$ : direct effect, $c$ : total effect, $* p<0.05, * * p<0.01, * * * p<0.001$. CTQ: Childhood Trauma Questionnaire.

\section{Discussion}

The present study examined the reliability and validity of a Chinese version (COHS-C) of the $\mathrm{COHS}$ and to validate the two-factorial structure of habitual behavior assessed by the COHS across different cultures. Generally, the Chinese version of $\mathrm{COHS}$ exhibited a good to excellent internal consistency reliability and confirmed the two-factorial structure of habitual behavior response patterns in daily life as assessed by self-report. Based on the previous literature, we hypothesized that higher exposure to ELS (before the age of 16) would associate with higher self-reported habitual behavior and lower cognitive flexibility. In the present dataset both hypotheses could be confirmed. Moreover, the major aim of the present study was to explore whether cognitive flexibility would mediate the association between higher ELS and higher levels of habitual behavior. Testing our a priori hypothesis using a mediation model confirmed that cognitive flexibility mediated the association between ELS and habitual tendencies in everyday life. Specifically, higher exposure to aversive experiences during childhood related to more habitual behavior including both, routine and automatic behavioral patterns, in adulthood and the association was totally mediated by impaired cognitive flexibility, especially a decreased ability to perceive selfcontrol over behaviors and environment, but not the ability to perceive and generate multiple solutions. These findings suggest interventions that can improve the control facet of cognitive flexibility, specifically the tendency to perceive difficult situations as controllable, 
may strengthen the resilience to habitual behavior and may shift disrupted balance towards flexible goal-oriented behavior following elevated levels of aversive early life experiences.

The confirmatory factor analysis demonstrated a moderate model fit, suggesting that the two-factorial solution of habitual behavior reported for the original COHS version (Ersche et al., 2017; Ersche et al., 2019) generalizes across populations with different socio-economic and cultural backgrounds. Furthermore, factor loadings of all items were larger than 0.3 indicating that all items contributed to their respective scales, replicating previous results obtained with the original COHS version (Ersche et al., 2019). Both Cronbach's alpha and McDonald's omega for the scales indicate a high internal consistency reliability. Although some model indices suggest a rather moderate fit, similar results for these indices were observed for the original COHS in Caucasian samples (Ersche et al., 2017; Ersche et al., 2019). Together, the findings from the COHS-C suggest that the Chinese version of the original instrument meets the research standards for psychometric scales and its usage in studies in Chinese populations and generally confirms overarching models proposing habit formation in a context-dependent framework (Robbins \& Costa, 2017; Wood \& Runger, 2016). In the present sample the routine and automaticity subscales exhibited a higher correlation than in the previous assessments (Ersche et al., 2017; Ersche et al., 2019). This subtle difference between the versions may suggest either subtle cultural differences or reflect an effect of aging. The Chinese participants in the present sample were younger than the Caucasian participants in the studies evaluating the original English COHS (Ersche et al., 2017; Ersche et al., 2019) and previous studies suggest that younger adults may undergo a period of stronger stimulus-response learning (Juncos-Rabadan, Pereiro, \& Facal, 2008; Mell et al., 2005) to support both routine and automatic behaviors based on context-dependent association.

Our mediation models reveal that higher exposure to childhood adversity was associated with increased habit formation in subsequent daily life. The observed association is in line with accumulating evidence from animal models and experimental studies in humans (DiasFerreira et al., 2009; Harms et al., 2018; Park, Lee, \& Chey, 2017; Schwabe \& Wolf, 2011), self-report measurements assessing individual variations in the use of habitual behavior in everyday life (Ersche et al., 2017), as well as accumulating evidence suggesting that current and chronic stress render individuals at an elevated risk for the development of mental disorders characterized by dysregulated habits such as substance dependence (Enoch, 2011; Uhart \& Wand, 2009). Habits can reduce cognitive load under stress and ELS-associated behavioral and neural changes have been considered as potential adaptive mechanism to a harmful environment which can become dysfunctional during later adult life and thus promote the development of mental disorders (Teicher \& Samson, 2016; Teicher et al., 2016; Uhart \& Wand, 2009). From a psychological perspective reliance on automatic or regular responses under stress and in the face of danger may have clear benefits and reduce the load on limited cognitive resources. However, excessive or chronic stress during early sensitive periods has been associated with impaired cognitive functions, including cognitive flexibility which may impede the ability to generate alternative choices and adaptive behavior in the context of changing environmental contexts (e.g. Harms et al., 2018; Stuart, 
Hinchcliffe, \& Robinson, 2019). Cognitive flexibility represents an important resource to adaptively cope with stressful life events (Murphy et al., 2012) and ELS-induced impairments in this domain may shift the balance towards automatic habitual behavior. The present findings suggest that cognitive flexibility totally mediates the association between ELS and habitual behaviors. Efficient cognitive flexibility relies on the integrity of the prefrontal cortex, which is thought to implement goal-directed control (Armbruster, Ueltzhoffer, Basten, \& Fiebach, 2012), particularly model-based learning, which has been demonstrated to protect against habit formation (Gillan, Otto, Phelps, \& Daw, 2015). Noteworthy not all aspects of cognitive flexibility totally mediated the association between ELS and habitual behavior, such that specifically the control facet of cognitive flexibility impacted both, routine and automatic habitual behavior. Although the alternatives facet of cognitive flexibility influenced the direct model path associated with habits it did not mediate the association between ELS and habits. Our model thus highlights that the perception of controllability in the face of difficult situations mediates the bias for habitual behavior and may reflect that high levels of stress during early life may lead to a persistent feeling of loss of control over the environment which negatively impacts adaptive flexible behavior in favor of habitual behavior. As Dennis and Vander Wal (2010) proposed a lack of cognitive flexibility could increase a maladaptive tendency to utilize and consider adaptive coping strategies. In line with previous experimental findings (Gillan et al., 2015; Harms et al., 2018; Wood \& Runger, 2016) the present self-report findings thus emphasize that ELS-associated deficiencies in cognitive control may aggravate habitual tendencies including both routine and automatic behaviors (Gillan et al., 2015; Harms et al., 2018; Wood \& Runger, 2016).

Interestingly, a previous study examining associations between the COHS facets and impulsivity and compulsivity found that routine tendencies have a positive association with compulsivity and negative association with impulsivity, in contrast to automaticity relying more strongly on impulsivity than compulsivity (Ersche et al., 2019). Together with the present findings this may indicate an association between the $\mathrm{COHS}$ scales and subclinical symptoms of abnormal compulsive and impulsive behavioral tendencies following elevated ELS which have recently gained increasing interest in disorders of substance use and dependence (Everitt \& Robbins, 2016) and obsessive-compulsive disorder (OCD) (Adams et al., 2018; Robbins, Vaghi, \& Banca, 2019). In line with these observations accumulating evidence suggests that dysregulated habit learning may represent an important pathological pathway that is central to disorders of compulsivity characterized by a cycle of repetitive maladaptive behavior, including substance dependence (Dickinson, Wood, \& Smith, 2002; Everitt \& Robbins, 2016) and OCD (Gillan \& Robbins, 2014; Robbins et al., 2019).

Although the present study focused on healthy participants, it was observed that higher exposure to aversive childhood experiences was associated with stronger implementation of rigid routines and automatic behavioral patterns in everyday life which were mediated by levels of cognitive flexibility. Despite the lack of apparent psychiatric symptoms in the present young healthy sample it is conceivable that stronger reliance on habitual behavior may represent a vulnerability factor for some mental disorders which may become apparent with aging or under re-current / chronic stress exposure during adulthood. Promoting 
cognitive flexibility may thus represent an opportunity for early intervention and has been demonstrated to be particularly efficient for children and young adolescents, particularly those with low baseline cognitive flexibility and elevated levels of ELS, e.g. due to rearing in institutional care contexts (Crone, Richard Ridderinkhof, Worm, Somsen, \& Van Der Molen, 2004).

Accumulating evidence from animal models and human experimental research indicates that the dopaminergic reward system and associated fronto-striatal circuits between specific subregions of the striatum and the prefrontal cortex, anterior cingulate cortex and amygdala undergo lasting neuroadaptations as a consequence of ELS (Teicher \& Samson, 2016; Teicher et al., 2016). These circuits partly overlap with ones mediating the effects of stress on the development of addiction as well as the effects of stress on the formation of habits (Taylor et al., 2014). Alterations in fronto-striatal circuits following ELS have been suggested to promote diminished anticipation and insensitivity to changes in outcome values as well as task switching and decision making and these functions are crucial for adaptive goal-directed behavior (Teicher et al., 2016; Wood \& Runger, 2016). Cognitive flexibility also relies on a network of distributed systems encompassing the prefrontal cortex, anterior cingulate cortex, and striatum (Leber, Turk-Browne, \& Chun, 2008; van Schouwenburg, O'Shea, Mars, Rushworth, \& Cools, 2012) and dopamine-mediated increases in cognitive flexibility appear to be neurally mediated by effects on fronto-striatal circuits (Stelzel, Fiebach, Cools, Tafazoli, \& D'Esposito, 2013). Stress promotes a more rapid transition to stimulus-response action patterns by inducing a shift to specific striatal circuits, particularly sensorimotor and dorsal striatum loops (Wood \& Runger, 2016). Together, these previous neuroimaging and pharmacological studies suggest that ELS-induced adaptations in fronto-striatal circuits may underlie the observed effects which are possibly mediated by deficient engagement of the prefrontal cortex which is important for cognitive flexibility rather than increased engagement of striatal regions that mediate habit formation. Additionally, ELS experience may induce an altered cortisol stress response during childhood, which impairs the prefrontal cortex and may then shift the brain to the striatum habit system. However, associations between individual variations in the COHS subscales and in structural and functional brain systems remain to be determined and these hypotheses require evaluation in future studies.

The present findings need to be considered in the context of some limitations. For instance, we included the COHS that mainly assesses general habitual tendencies in daily life, but due to the low prevalence of drug use in comparable samples maladaptive habits, e.g. smoking, drug and alcohol abuse were not assessed. Thus, conclusions regarding direct effects of ELS on problematic behaviors remain limited, although the present investigation still allows insights on potential underlying pathways that mediate the detrimental effects of ELS on maladaptive behavioral tendencies. In addition, cross-validations of the associations should include participants from populations with higher diversity to further validate the generalizability as well as potential cross-cultural differences in habitual behavioral tendencies. Finally, the present study is a cross-sectional, correlational study based on selfreported measurement with no causality. 


\section{Conclusions}

In the present study we developed and evaluated a Chinese version of the Creature of Habits Scale (COHS) and applied a mediation approach to examine whether the association between exposure to aversive childhood experience and stronger habitual behavior in adulthood is mediated by cognitive flexibility. The Chinese version of the COHS (COHS-C) exhibited good to excellent psychometric properties and confirmed the two-factor structure observed for the original version. In line with our hypothesis cognitive flexibility mediated the association between early life stress and habitual behavior.

\section{Acknowledgements}

This work was supported by the National Key Research and Development Program of China (Grant No. 2018YFA0701400), the National Natural Science Foundation of China (NSFC, 91632117 to BB), the Sichuan Science and Technology Department (2018JY0001 to BB). 


\section{References}

Adams, T. G., Kelmendi, B., Brake, C. A., Gruner, P., Badour, C. L., \& Pittenger, C. (2018). The role of stress in the pathogenesis and maintenance of obsessive-compulsive disorder. Chronic Stress (Thousand Oaks), 2. doi:10.1177/2470547018758043

Armbruster, D. J., Ueltzhoffer, K., Basten, U., \& Fiebach, C. J. (2012). Prefrontal cortical mechanisms underlying individual differences in cognitive flexibility and stability. $J$ Cogn Neurosci, 24(12), 2385-2399. doi:10.1162/jocn_a_00286

Beaton, D. E., Bombardier, C., Guillemin, F., \& Ferraz, M. B. (2000). Guidelines for the process of cross-cultural adaptation of self-report measures. Spine (Phila Pa 1976), 25(24), 3186-3191. doi:10.1097/00007632-200012150-00014

Bernstein, D. P., Stein, J. A., Newcomb, M. D., Walker, E., Pogge, D., Ahluvalia, T., . . Zule, W. (2003). Development and validation of a brief screening version of the Childhood Trauma Questionnaire. Child Abuse Negl, 27(2), 169-190. doi:10.1016/s01452134(02)00541-0

Bonapersona, V., Joels, M., \& Sarabdjitsingh, R. A. (2018). Effects of early life stress on biochemical indicators of the dopaminergic system: A 3 level meta-analysis of rodent studies. Neurosci Biobehav Rev, 95, 1-16. doi:10.1016/j.neubiorev.2018.09.003

Claessens, S. E., Daskalakis, N. P., van der Veen, R., Oitzl, M. S., de Kloet, E. R., \& Champagne, D. L. (2011). Development of individual differences in stress responsiveness: an overview of factors mediating the outcome of early life experiences. Psychopharmacology, 214(1), 141-154.

Cronbach, L. J. (1951). Coefficient alpha and the internal structure of tests. psychometrika, 16(3), 297-334.

Crone, E. A., Richard Ridderinkhof, K., Worm, M., Somsen, R. J., \& Van Der Molen, M. W. (2004). Switching between spatial stimulus-response mappings: a developmental study of cognitive flexibility. Developmental science, 7(4), 443-455.

Daskalakis, N. P., Bagot, R. C., Parker, K. J., Vinkers, C. H., \& de Kloet, E. R. (2013). The three-hit concept of vulnerability and resilience: toward understanding adaptation to early-life adversity outcome. Psychoneuroendocrinology, 38(9), 1858-1873.

Dennis, J. P., \& Vander Wal, J. S. (2010). The cognitive flexibility inventory: Instrument development and estimates of reliability and validity. Cognitive therapy and research, 34(3), 241-253.

Dias-Ferreira, E., Sousa, J. C., Melo, I., Morgado, P., Mesquita, A. R., Cerqueira, J. J., . . Sousa, N. (2009). Chronic stress causes frontostriatal reorganization and affects decisionmaking. Science, 325(5940), 621-625. doi:10.1126/science.1171203

Dickinson, A., Wood, N., \& Smith, J. W. (2002). Alcohol seeking by rats: action or habit? Q J Exp Psychol B, 55(4), 331-348. doi:10.1080/0272499024400016

Enoch, M.-A. (2011). The role of early life stress as a predictor for alcohol and drug dependence. Psychopharmacology, 214(1), 17-31.

Ersche, K. D., Gillan, C. M., Jones, P. S., Williams, G. B., Ward, L. H., Luijten, M., . . Robbins, T. W. (2016). Carrots and sticks fail to change behavior in cocaine addiction. Science, 352(6292), 1468-1471. doi:10.1126/science.aaf3700

Ersche, K. D., Lim, T. V., Ward, L. H. E., Robbins, T. W., \& Stochl, J. (2017). Creature of Habit: A self-report measure of habitual routines and automatic tendencies in everyday life. 
Pers Individ Dif, 116, 73-85. doi:10.1016/j.paid.2017.04.024

Ersche, K. D., Ward, L. H. E., Lim, T. V., Lumsden, R. J., Sawiak, S. J., Robbins, T. W., \& Stochl, J. (2019). Impulsivity and compulsivity are differentially associated with automaticity and routine on the Creature of Habit Scale. Pers Individ Dif, 150, 109493. doi:10.1016/j.paid.2019.07.003

Everitt, B. J., \& Robbins, T. W. (2016). Drug Addiction: Updating Actions to Habits to Compulsions Ten Years On. Annu Rev Psychol, 67, 23-50. doi:10.1146/annurev-psych122414-033457

Gibb, B. E., Schofield, C. A., \& Coles, M. E. (2009). Reported history of childhood abuse and young adults' information-processing biases for facial displays of emotion. Child maltreatment, 14(2), 148-156.

Gillan, C. M., Otto, A. R., Phelps, E. A., \& Daw, N. D. (2015). Model-based learning protects against forming habits. Cogn Affect Behav Neurosci, 15(3), 523-536. doi:10.3758/s13415-015-0347-6

Gillan, C. M., \& Robbins, T. W. (2014). Goal-directed learning and obsessive-compulsive disorder. Philos Trans R Soc Lond B Biol Sci, 369(1655). doi:10.1098/rstb.2013.0475

Harms, M. B., Shannon Bowen, K. E., Hanson, J. L., \& Pollak, S. D. (2018). Instrumental learning and cognitive flexibility processes are impaired in children exposed to early life stress. Dev Sci, 21(4), e12596. doi:10.1111/desc.12596

Huang, H. W., Hsueh, K. C., Lu, C. C., \& Huang, C. L. (2016). Psychometric Evaluation of the Cigarette Withdrawal Scale (Chinese Version) in Male Smokers in Taiwan. J Nurs Res, 24(2), 118-125. doi:10.1097/jnr.0000000000000113

Juncos-Rabadan, O., Pereiro, A. X., \& Facal, D. (2008). Cognitive interference and aging: insights from a spatial stimulus-response consistency task. Acta Psychol (Amst), 127(2), 237246. doi:10.1016/j.actpsy.2007.05.003

Klugah-Brown, B., Di, X., Zweerings, J., Mathiak, K., Becker, B., \& Biswal, B. (2020). Common and separable neural alterations in substance use disorders: evidence from coordinate-based meta-analyses of functional neuroimaging studies in human. bioRxiv.

Leber, A. B., Turk-Browne, N. B., \& Chun, M. M. (2008). Neural predictors of moment-tomoment fluctuations in cognitive flexibility. Proceedings of the National Academy of Sciences, 105(36), 13592-13597.

Liu, C., Xu, L., Li, J., Zhou, F., Yang, X., Zheng, X., .. Montag, C. (2019). Serotonin and childhood maltreatment interact to shape brain architecture and anxious avoidant behavior-a TPH2 imaging genetics approach. bioRxiv, 685099.

McDonald, R. P. (2013). Test theory: A unified treatment: psychology press.

Meade, A. W., \& Craig, S. B. (2012). Identifying careless responses in survey data. Psychol Methods, 17(3), 437-455. doi:10.1037/a0028085

Mell, T., Heekeren, H. R., Marschner, A., Wartenburger, I., Villringer, A., \& Reischies, F. M. (2005). Effect of aging on stimulus-reward association learning. Neuropsychologia, 43(4), 554563. doi:10.1016/j.neuropsychologia.2004.07.010

Murphy, F. C., Michael, A., \& Sahakian, B. J. (2012). Emotion modulates cognitive flexibility in patients with major depression. Psychol Med, 42(7), 1373-1382. doi:10.1017/S0033291711002418 
Nunnally, J. C. (1994). Psychometric theory 3E: Tata McGraw-Hill Education.

Park, H., Lee, D., \& Chey, J. (2017). Stress enhances model-free reinforcement learning only after negative outcome. PLoS One, 12(7), e0180588. doi:10.1371/journal.pone.0180588

Patterson, T., Craske, M., \& Knowlton, B. J. (2019). Enhanced avoidance habits in relation to history of early-life stress. Frontiers in psychology, 10, 1876.

Peters, M., \& Passchier, J. (2006). Translating instruments for cross-cultural studies in headache research. Headache, 46(1), 82-91. doi:10.1111/j.1526-4610.2006.00298.x

Podsakoff, P. M., MacKenzie, S. B., Lee, J. Y., \& Podsakoff, N. P. (2003). Common method biases in behavioral research: a critical review of the literature and recommended remedies. J Appl Psychol, 88(5), 879-903. doi:10.1037/0021-9010.88.5.879

Preacher, K. J., \& Hayes, A. F. (2004). SPSS and SAS procedures for estimating indirect effects in simple mediation models. Behav Res Methods Instrum Comput, 36(4), 717-731. doi:10.3758/bf03206553

Preacher, K. J., \& Hayes, A. F. (2008). Asymptotic and resampling strategies for assessing and comparing indirect effects in multiple mediator models. Behav Res Methods, 40(3), 879-891. doi:10.3758/brm.40.3.879

Puzia, M. E., Kraines, M. A., Liu, R. T., \& Kleiman, E. M. (2014). Early life stressors and suicidal ideation: Mediation by interpersonal risk factors. Personality and Individual Differences, 56, 68-72.

Robbins, T. W., \& Costa, R. M. (2017). Habits. Curr Biol, 27(22), R1200-R1206. doi:10.1016/j.cub.2017.09.060

Robbins, T. W., Vaghi, M. M., \& Banca, P. (2019). Obsessive-compulsive disorder: puzzles and prospects. Neuron, 102(1), 27-47.

Rutter, M., Kumsta, R., Schlotz, W., \& Sonuga-Barke, E. (2012). Longitudinal studies using a "natural experiment" design: the case of adoptees from Romanian institutions. Journal of the American Academy of Child \& Adolescent Psychiatry, 51(8), 762-770.

Schwabe, L., \& Wolf, O. T. (2011). Stress-induced modulation of instrumental behavior: from goal-directed to habitual control of action. Behav Brain Res, 219(2), 321-328. doi:10.1016/j.bbr.2010.12.038

Sheridan, M. A., McLaughlin, K. A., Winter, W., Fox, N., Zeanah, C., \& Nelson, C. A. (2018). Early deprivation disruption of associative learning is a developmental pathway to depression and social problems. Nature communications, 9(1), 1-8.

Simpson, J. A., Griskevicius, V., Kuo, S. I., Sung, S., \& Collins, W. A. (2012). Evolution, stress, and sensitive periods: the influence of unpredictability in early versus late childhood on sex and risky behavior. Developmental psychology, 48(3), 674.

Sonuga-Barke, E. J. S., Kennedy, M., Kumsta, R., Knights, N., Golm, D., Rutter, M., . . Kreppner, J. (2017). Child-to-adult neurodevelopmental and mental health trajectories after early life deprivation: the young adult follow-up of the longitudinal English and Romanian Adoptees study. Lancet, 389(10078), 1539-1548. doi:10.1016/S01406736(17)30045-4

Stelzel, C., Fiebach, C. J., Cools, R., Tafazoli, S., \& D'Esposito, M. (2013). Dissociable frontostriatal effects of dopamine D2 receptor stimulation on cognitive versus motor flexibility. Cortex, 49(10), 2799-2811. 
Stuart, S. A., Hinchcliffe, J. K., \& Robinson, E. S. (2019). Evidence that neuropsychological deficits following early life adversity may underlie vulnerability to depression. Neuropsychopharmacology, 44(9), 1623-1630.

Taylor, S. B., Anglin, J. M., Paode, P. R., Riggert, A. G., Olive, M. F., \& Conrad, C. D. (2014). Chronic stress may facilitate the recruitment of habit- and addiction-related neurocircuitries through neuronal restructuring of the striatum. Neuroscience, 280, 231-242. doi:10.1016/j.neuroscience.2014.09.029

Teicher, M. H., \& Samson, J. A. (2016). Annual Research Review: Enduring neurobiological effects of childhood abuse and neglect. J Child Psychol Psychiatry, 57(3), 241-266. doi:10.1111/jcpp.12507

Teicher, M. H., Samson, J. A., Anderson, C. M., \& Ohashi, K. (2016). The effects of childhood maltreatment on brain structure, function and connectivity. Nat Rev Neurosci, 17(10), 652-666. doi:10.1038/nrn.2016.111

Ten Have, M., de Graaf, R., van Dorsselaer, S., Tuithof, M., Kleinjan, M., \& Penninx, B. (2019). Childhood maltreatment, vulnerability characteristics and adult incident common mental disorders: 3-year longitudinal data among $>10,000$ adults in the general population. J Psychiatr Res, 113, 199-207. doi:10.1016/j.jpsychires.2019.03.029

Uhart, M., \& Wand, G. S. (2009). Stress, alcohol and drug interaction: an update of human research. Addict Biol, 14(1), 43-64. doi:10.1111/j.1369-1600.2008.00131.x

van Schouwenburg, M. R., O'Shea, J., Mars, R. B., Rushworth, M. F., \& Cools, R. (2012). Controlling human striatal cognitive function via the frontal cortex. Journal of Neuroscience, 32(16), 5631-5637.

Wang, Y., Yang, Y., Xiao, W.-T., \& Su, Q. (2016). Validity and reliability of the Chinese version of the Cognitive Flexibility Inventory in college students. Chinese mental health journal.

Wood, W., \& Runger, D. (2016). Psychology of Habit. Annu Rev Psychol, 67, 289-314. doi:10.1146/annurev-psych-122414-033417

Wu, J., Yuan, M., \& Kou, Y. (2020). Disadvantaged early-life experience negatively predicts prosocial behavior: The roles of Honesty-Humility and dispositional trust among Chinese adolescents. Personality and Individual Differences, 152, 109608.

Zhao, X. F., Zhang, Y. L., Li, L. F., Zhou, Y. F., Li, H. Z., Yang, S. C., .. Province, H. (2005). Reliability and validity of the Chinese version of childhood trauma questionnaire. Chinese Journal of Clinical Rehabilitation, 9(20), 105-107.

Zhou, X., Zimmermann, K., Xin, F., Zhao, W., Derckx, R. T., Sassmannshausen, A., . . Becker, B. (2019). Cue Reactivity in the Ventral Striatum Characterizes Heavy Cannabis Use, Whereas Reactivity in the Dorsal Striatum Mediates Dependent Use. Biological Psychiatry: Cognitive Neuroscience and Neuroimaging, 4(8), 751-762. 


\section{Supplementary materials}

Table S1. Items of COHS-C

1. 我喜欢把我的汽车/自行车/电动车/摩托车停在同样的地方。

2. 我一般用同样的调料/口味做饭。

3. 当经过一盘糖果或饼干时, 我会忍不住拿一块。

4. 我倾向于每天晚上在差不多同一时间睡觉。

5. 我经常在(例如开车、逛街或上网时)吃点零食。

6. 如果没有必要, 我很乐意在自己的舒适区工作, 而不是挑战自己。

7. 我倾向于每天早上按同样的顺序做事(例如起床、上则所、喝水……) 。

8. 我一般直接从包装袋里吃薯片或饼干。

9. 每当我走进厨房, 我通常会看看冰箱。

10. 我总是设法在公共汽车上、电影院、教室等地方找到同一个座位/位置。

11. 我经常发现自己吃完一包饼干只是因为它就放在那里。

12. 我通常在同一家超市/商店买同样的食物。

13. 我依赖那些已经尝试和测试过的事物, 而不是探索新的事物。

14. 我一般每天早餐吃同样的东西。

15. 我比较喜欢遵循惯例。

16. 我通常在日常工作结束时给自己吃点零食。

17. 在餐馆里, 我倾向于点我熟悉的菜。

18. 我是那种对在最后一刻时取消计划/安排感到很恼火的人。

19. 我经常发现自己不知不觉就在吃东西。

20. 我通常坐在餐桌的同一位置。

21. 我经常发现自己在 “自动行动” ，然后会疑惑为什么我最终停在一个特定的地 方或做一些我不打算做的事情。

22. 我总是按照一定的顺序准备一顿饭。

23. 看电视使我特别容易不受控制地进食。

24. 我倾向于尽可能长时间地坚持使用我熟悉的软件版本。

25. 我经常发现自己打开蚞柜/冰箱吃零食。

26. 当我感到压力时, 我很容易吃得更多。

27. 我在规律性中感到舒适安心。 